\title{
586.
}

\section{ON THE MATHEMATICAL THEORY OF ISOMERS.}

[From the Philosophical Magazine, vol. xLvII. (1874), pp. 444-446.]

I CONSIDER a "diagram," viz. a set of points $H, O, N, C$, \&c. (any number of each), connected by links into a single assemblage under the condition that through each $H$ there passes not more than one link, through each $O$ not more than two links, through each $N$ not more than three links, through each $C$ not more than four links. Of course through every point there passes at least one link, or the points would not be connected into a single assemblage.

In such a diagram each point having its full number of links is saturate, or nilvalent: in particular, each point $H$ is saturate. A point not having its full number of links is univalent, bivalent, or trivalent, according as it wants one, two, or three of its full number of links. If every point is saturate the diagram is saturate, or nilvalent; or, say, it is a "plerogram"; but if the diagram is susceptible of $n$ more links, then it is $n$-valent; viz. the valency of the diagram is the sum of the valencies of the component points.

Since each $H$ is connected by a single link (and therefore to a point $O, C, \&$ c. as the case may be, but not to another point $H$ ), we may without breaking up the diagram remove all the points $H$ with the links belonging to them, and thus obtain a diagram without any points $H$ : such a diagram may be termed a "kenogram": the valency is obviously that of the original diagram plus the number of removed $H$ 's.

If from a kenogram, we remove every point $O, C, \& c$. connected with the rest of the diagram by a single link only (each with the link belonging to it), and so on indefinitely as long as the process is practicable, we arrive at last at a diagram in which every point $O, C$, \&c. is connected with the rest of the diagram by two links at least: this may be called a "mere kenogram." 
Each or any point of a mere kenogram may be made the origin of a "ramification"; viz. we have here links branching out from the original point, and then again from the derived points, and so on any number of times, and never again uniting. We can thus from the mere kenogram obtain (in an infinite variety of ways) a diagram. The diagram completely determines the mere kenogram; and consequently two diagrams cannot be identical unless they have the same mere kenogram. Observe that the mere kenogram may evanesce altogether; viz. this will be the case if the diagram or kenogram is a simple ramification.

A ramification of $n$ points $C$ is $(2 n+2)$-valent: in fact, this is so in the most simple case $n=1$; and admitting it to be true for any value of $n$, it is at once seen to be true for the next succeeding value. But no kenogram of points $C$ is so much as $(2 n+2)$-valent; for instance, 3 points $C$ linked into a triangle, instead of being 8 -valent are only 6 -valent. We have therefore plerograms of $n$ points $C$ and $2 n+2$ points $H$, say plerograms $C H^{2 n+2}$; and in any such plerogram the kenogram is of necessity a ramification of $n$ points $C$; viz. the different cases of such ramifications are *

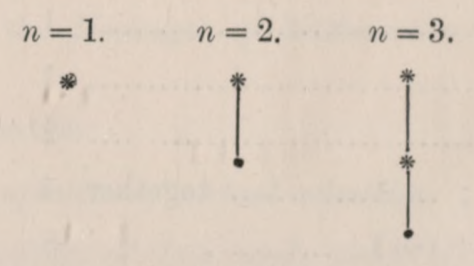

$(\alpha)$

$(\alpha)$ $(\alpha)$

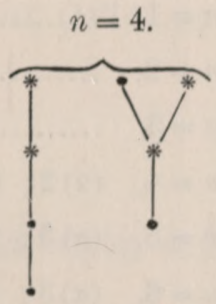

$(\alpha)$ $(\beta)$

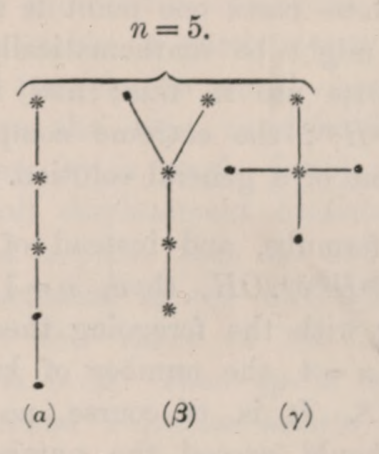

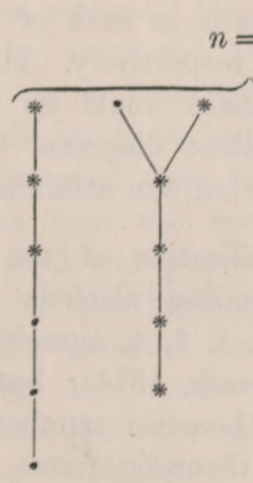

$(\beta)$

$n=6$.

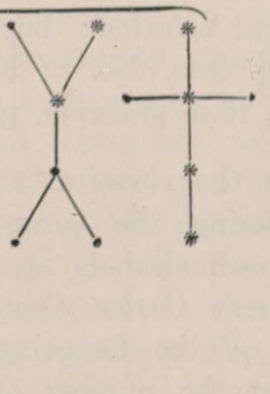

$(\gamma)$
$(8)$

where the mathematical question of the determination of such forms belongs to the class of questions considered in my paper "On the Theory of the Analytical Forms called Trees," Phil. Mag. vol. xIII. (1857), [203], and vol. xvIII. (1859), [247], and in some papers on Partitions in the same Journal.

* The distinction in the diagrams of asterisks and dots is to be in the first instance disregarded; it is made in reference to what follows, the explanation as to the allotrious points.

$26-2$ 
The different forms of univalent diagrams $C^{n} H^{2 n+1}$ are obtained from the same ramifications by adding to each of them all but one of the $2 n+2$ points $H$; that is, by adding to each point $C$ except one its full number of points $H$, and to the excepted point one less than the full number of points $H$. The excepted point $C$ must therefore be univalent at least; viz. it cannot be a saturate point, which presents itself for example in the diagrams $n=5(\gamma)$ and $n=6(\delta)$. And in order to count the number of distinct forms (for the diagrams $C^{n} H^{2 n+1}$ ), we must in each of the above ramifications consider what is the number of distinct classes into which the points group themselves, or, say, the number of "allotrious" points. For instance, in the ramification $n=3$ there are two classes only; viz. a point is either terminal or medial; or, say, the number of allotrious points is $=2$ : this is shown in the diagrams by means of the asterisks; so that in each case the points which may be considered allotrious are represented by asterisks, and the number of asterisks is equal to the number of allotrious points.

Thus, number of univalent diagrams $C^{n} H^{2 n+1}$ :

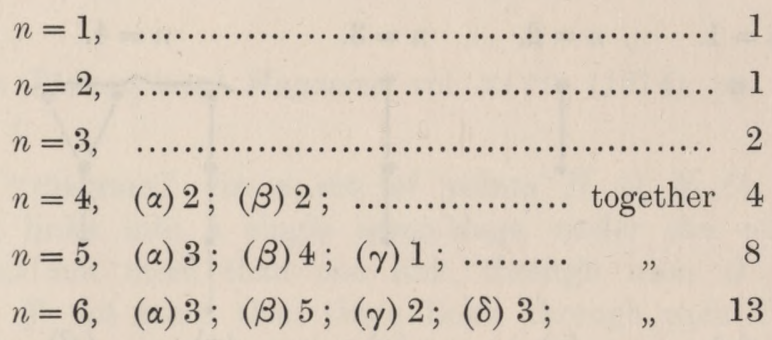

where it will be observed that, $n=5(\gamma)$, and $n=6(\delta)$, the numbers of allotrious points are 2 and 4 respectively; but since in each of these cases one point is saturate, they give only the numbers 1 and 3 respectively. It might be mathematically possible to obtain a general solution; but there would be little use in this; and for even the next succeeding case, No. of bivalent diagrams $C^{n} H^{2 n}$; the extreme complexity of the question would, it is probable, prevent the attainment of a general solution.

Passing to the chemical signification of the formulæ, and instead of the radicals $C^{n} H^{2 n+1}$ considering the corresponding alcohols $C^{n} H^{2 n+1} . O H$, then, $n=1,2,3,4$, the numbers of known alcohols are 1, 1, 2, 4, agreeing with the foregoing theoretic number (see Schorlemmer's Carbon Compounds, 1874); but $n=4$, the number of known alcohols is $=2$, instead of the foregoing theoretic number 8 . It is, of course, no objection to the theory that the number of theoretic forms should exceed the number of known compounds; the missing ones may be simply unknown; or they may be only capable of existing under conceivable, but unattained, physical conditions (for instance, of temperature); and if defect from the theoretic number of compounds can be thus accounted for, the theory holds good without modification. But it is also possible that the diagrams, in order that they may represent chemical compounds, may be subject to some as yet undetermined conditions; viz. in this case the theory would stand good as far as it goes, but wonld require modification. 